\title{
Território de saúde: possibilidades e desafios a partir da contribuição de Norbert Elias e John L. Scotson
}

\author{
Health territory: possibilities and challenges from the \\ contribution of Norbert Elias and John L. Scotson
}

Cintya Cristine Martins da Veiga Faria ${ }^{1 *}$ (D), Carlos Henrique Assunção Paiva² (D)

\begin{abstract}
Resumo
Introdução: As discussões sobre Saúde e Território têm chamado a atenção para o necessário envolvimento da sociedade em uma agenda de mudanças e de apoio ao Sistema Único de Saúde (SUS), inclusive para as diferentes relações estabelecidas entre equipes de saúde e população. A realidade vivenciada pelas equipes de saúde e população demonstra que possíveis problemas inerentes a certos desafios acabam por comprometer não apenas a relação entre uma e outra, mas igualmente a proposta de universalização da saúde. Objetivo: Contemplar a discussão sobre Saúde e Território a partir da contribuição de Norbert Elias e John L. Scotson, autores que, a partir da perspectiva das configurações territoriais, trazem importantes aportes para os debates da Saúde Coletiva. Pretende-se discutir as questões de natureza sociológica na composição de relações de poder e de sociabilidade no interior do território, destacando-se a problemática das relações entre indivíduos em uma comunidade e visando, assim, identificar as relações de poder, dependência e exclusão existentes em um território específico. Método: Foi realizada uma revisão não sistemática da literatura especializada, definida de acordo com aproximações conceituais que fossem capazes de informar o debate em torno do binômio território e saúde. Assim, pretende-se apreender e compreender a existência de grupos e subgrupos muitas vezes ignorados no conjunto das relações efetivas. Resultados: Os resultados apontam para a necessidade de uma reavaliação das relações díspares na organização de uma sociedade, bem como seus efeitos na vida dos habitantes. As respostas apresentadas revelam concepções rígidas de pensamento e comportamento que diferenciam e isolam o indivíduo da sociedade. Há urgência de ferramentas para uma melhor percepção dos desafios e possibilidades de atuação dos profissionais nos territórios de saúde, principalmente no que se refere à noção de território. Conclusão: São muitos os obstáculos enfrentados tanto pela gestão da Atenção Primária à Saúde, quanto pela assistência do cuidado. Não basta considerar de forma singular os aspectos do comportamento ou das ações dos indivíduos, pois é preciso reavaliar as configurações envolvidas na interdependência estabelecida entre as pessoas dentro de um território.
\end{abstract}

Palavras-chave: território de saúde; atenção primária à saúde; configurações sociais; comunidade; recursos humanos em saúde.

\footnotetext{
Abstract

Background: The discussions about Health and Territory have drawn attention to the necessary involvement of society regarding the structural changes of the Unified Health System (SUS), including the different relationships between health teams and the population. The reality experienced by the health teams and the population shows that possible problems inherent to the challenges end up compromising not only the relationship between them, but also the proposal of universalization of health. Objective: Contemplate

1Programa de Pós-graduação em Saúde da Família, Universidade Estácio de Sá (UNESA) - Rio de Janeiro (RJ), Brasil.

²Observatório História e Saúde, Centro de Documentação e História da Saúde, Casa de Oswaldo Cruz (COC), Fundação Oswaldo Cruz (FIOCRUZ) - Rio de Janeiro (RJ), Brasil.

Trabalho realizado no Programa de Pós-graduação em Saúde da Família, Universidade Estácio de Sá - Rio de Janeiro (RJ), Brasil.

Endereço para correspondência: Carlos Henrique Assunção Paiva - Observatório História e Saúde, Centro de Documentação e História da Saúde, Casa de Oswaldo Cruz (COC), Fundação Oswaldo Cruz (FIOCRUZ), Avenida Brasil, 4365, Sala 315 - Manguinhos - CEP: 21040-900 - Rio de Janeiro (RJ), Brasil Email: chapaiva@gmail.com

Fonte de financiamento: nenhuma.

Conflito de interesses: nada a declarar.
}

Este é um artigo publicado em acesso aberto (Open Access) sob a licença Creative Commons Attribution, que permite uso, distribuição e reprodução em qualquer meio, sem restrições desde que o trabalho original seja corretamente citado. 
the discussion about Health and Territory, from the contributions of Norbert Elias and John L. Scotson, authors that, from the perspective of social configurations, bring important contributions for Public Health debates. It is intended to discuss sociological issues in the composition of power relations and sociability within the territory, highlighting the problematic of relationships between individuals in a Community, thus, aiming to identify power relations, dependency and exclusion in a specific territory. Method: A non-systematic review of the specialized literature was performed, defined according to conceptual approaches that were able to inform the debate around the territory and health binomial. Thus, it is intended to apprehend and understand the existence of groups and subgroups often ignored in the set of effective relationships. Purpose: consider the discussion on Health and Territory from the contribution of Norbert Elias and John L. Scotson, authors whose, from the perspective of territorial configurations, bring important contributions to the Collective Health debates. It is intended to discuss sociological issues in the composition of relations of power and sociability within the territory, highlighting the problematic of the relations between individuals in a community; thus, aiming to identify the relations of power, dependence and exclusion that exist in a specific territory. Results: The outcomes point to the need for a reassessment of disparate relationships in the organization of a Society, as well as their effects on the lives of the inhabitants. The answers presented to reveal rigid conceptions of thought and behavior that differentiate and isolate the individual from society. There is an urgent need for tools to better understand the challenges and possibilities for professionals to act in Health Territories, especially regarding the notion of the territory. Conclusion: There are many obstacles faced both by the management of Primary Health Care, as by care assistance. It is not enough to consider singularly the aspects of behavior or actions of individuals, because it is necessary to reassess the configurations involved in the interdependence established between people within a territory. Keywords: health territory; primary health care; social configurations; community; human resources in health.

\section{INTRODUÇÃO}

É ampla a literatura especializada que aborda as discussões sobre Saúde e Território. Para Gondim e Monken ${ }^{1}$, algumas dessas discussões comprometem-se com o envolvimento da sociedade no que se refere às mudanças estruturais do Sistema Único de Saúde (SUS) representando, portanto, contribuições relevantes para o debate sobre cidadania e seguridade social no país.

Desde a promulgação da Lei Orgânica da Saúde (1990), que marcou uma intencionalidade para uma nova abordagem de assistência à saúde da população, são pensadas iniciativas de intervenção concreta na realidade cotidiana dos espaços sociais que dialogam diretamente com as práticas sanitárias ${ }^{2}$. Neste sentido, como nos chamam a atenção Baralhas e Pereira ${ }^{3}$, é que se inserem as relações estabelecidas entre equipes de saúde e população, bem como os principais desafios enfrentados nessa mesma direção.

A realidade vivenciada pelas equipes de saúde e população demonstra que possíveis problemas inerentes a tais desafios acabam por comprometer não apenas a relação entre uma e outra, mas igualmente a proposta de universalização da saúde, que deve ofertar ações de saúde a um território heterogêneo e marcado por diferentes configurações sociais ${ }^{4}$.

Para Baralhas e Pereira ${ }^{3}$, boa parte dos estudos, no que concerne à chave saúde e território, centrou-se em questões relativas à noção de território na perspectiva de sua organização e nas suas relações com as práticas profissionais, especialmente no campo da Atenção Primária à Saúde (APS) / Estratégia Saúde da Família (ESF). Nessa linha, a perspectiva - por assim dizer, dominante - procura enfrentar o problema da organização dos processos por meio das práticas de trabalho e das práticas de saúde, uma vez que as ações de saúde são implementadas sobre uma base territorial.
Gondim e colaboradores ${ }^{1}$, por exemplo, trazem o conceito de espaços como conjuntos de territórios e lugares nos quais fatos acontecem simultaneamente. As repercussões desses fatos, tais como violência, desemprego, baixa escolaridade, falta de acesso aos serviços sociais e o pluralismo nos cuidados de saúde, são sentidas em sua totalidade de maneiras diferentes.

Ainda de acordo com Gondim e colaboradores ${ }^{1}$, o território de saúde como espaço vem sendo utilizado com ênfase no campo da saúde como uma abordagem fundamental para dar suporte ao conceito de risco. Isso se deve em função das múltiplas possibilidades de se localizarem e visualizarem populações, objetos e fluxos, além de se espacializar a situação de saúde por meio da distribuição de indicadores socioeconômicos, sanitários e ambientais que revelam as condições de vida das pessoas no interior de um determinado território de saúde.

Em que pese a existência de estudos importantes nesta discussão, não podemos deixar de observar certa reticência no que tange às questões de natureza sociológica na composição de relações de poder e de sociabilidade no interior do território espacial, e, surpreendentemente, em um campo cuja origem é marcada pela interdisciplinaridade e diversidade de enfoques, como é o caso da Saúde Coletiva.

Tendo em vista esse cenário de questões, o objetivo deste artigo é refletir sobre a potencial contribuição de Elias e Scotson ${ }^{4}$ para as análises da saúde coletiva que se ocupam do território de atuação de profissionais de saúde e da abrangência de serviços de saúde.

\section{TERRITÓRIO E COMUNIDADE}

Ao considerarmos as iniciativas de intervenção concreta na realidade cotidiana dos espaços sociais, não podemos deixar de considerar que a ideia de intervenção social dialoga diretamente com as práticas sanitárias. Estas, por sua vez, são permeadas por 
relações institucionais e sociais complexas, inclusive expressas no cotidiano humano vivenciado em diferentes territórios.

Não obstante, Santos e Rigotto ${ }^{5}$ destacam que os profissionais do SUS vêm tratando a operacionalização da categoria "território" de forma parcial, de maneira que o conceito de espaço finda por ser empregado a fins administrativos e voltados para o aspecto gerencial dos serviços de saúde. Tal dinâmica tem limitado o potencial e as possibilidades quanto à identificação de demandas de saúde, assim como nas formas de intervenção na realidade cotidiana das coletividades humanas.

Para além da proporção político-operativa do sistema de saúde, Santos e Rigotto ${ }^{5}$ acentuam que o território, na condição de cotidiano vivido, no qual acontece a interação entre as pessoas e os serviços de saúde no nível local do SUS, caracteriza-se por abranger uma população específica, vivendo em tempo e espaço determinados, com problemas de má saúde definidos, mas quase sempre com condicionantes e determinantes que despontam de um plano mais geral. Para os autores, esse território apresenta um cenário histórico, demográfico, epidemiológico, administrativo, político, social e cultural que o tipifica como um território em permanente construção.

Tal dimensão, de acordo com Monken e Barcelos ${ }^{2}$, convoca a atenção para a precariedade desta noção administrativa de território que distancia as ações da realidade e impedem a perspectiva da participação social.

Eleger a participação social no território de saúde determina que se compreenda a existência de configurações sociais específicas, a interdependência e o equilíbrio de tensões, pertencentes à própria comunidade.

Ao incorporarmos os argumentos dos pesquisadores citados, remetemos aos conceitos de Elias e Scotson ${ }^{4}$ sobre tais aspectos, quando afirmam que os indivíduos estão relacionados uns aos outros dentro de uma pluralidade, isto é, numa sociedade.

Para Elias e Scotson ${ }^{4}$, a problemática do relacionamento entre os indivíduos em uma comunidade é identificada e elucidada a partir de como se estabelecem as relações de poder, dependência e exclusão, existentes em uma sociedade específica, e suas implicações em todos os níveis de sua organização, bem como em todos os aspectos da vida dos habitantes.

No que se refere ao entendimento das ciências sociais e da saúde coletiva, de acordo com Nascimento et al. ${ }^{6}$, o trabalho de Norbert Elias e Scotson ${ }^{4}$ apresenta, em suas concepções, uma riqueza de possibilidades e força conceitual para definir a elaboração tanto da pesquisa quanto da relação entre ciências sociais e saúde coletiva.

Desse modo, ao nos apropriarmos das concepções eliasianas sobre configurações sociais específicas, utilizando-as para as intervenções no território de saúde, reforçamos o potencial de gerar tanto um maior envolvimento da sociedade no processo de mudanças estruturais do SUS quanto uma análise das relações estabelecidas entre os atores sociais envolvidos. Assim, como já instituído, legitima-se a intencionalidade para uma nova abordagem de assistência à saúde.

\section{EQUIPES DE SAÚDE E DIVERSIDADE SOCIAL: O DESAFIO DE UMA RELAÇÃO DIALÓGICA}

Ao pesquisarmos as diferentes concepções identificadas na obra de Elias e Scotson ${ }^{4}$, no que se refere a aspectos figuracionais encontrados em seu campo de pesquisa, torna-se imprescindível analisar que tais aspectos são capazes de estabelecer uma condição hierárquica estigmatizante e excludente, assim como podem ser percebidos em muitos outros contextos sociais.

Ao destacarem, em sua obra, a problematização das relações entre os indivíduos em uma sociedade específica, os autores nos falam de uma dinâmica que transcende as normas nas relações de poder, dependência e exclusão, que se estabelecem dentro de um determinado grupo.

Esse processo tem suas implicações em todos os níveis de organização dessa coletividade, assim como em todos os aspectos da vida de seus habitantes.

Na perspectiva da Saúde Coletiva, os aspectos figuracionais que se apresentam nos territórios de saúde podem estabelecer uma perigosa estrutura hierárquica, dificultando o acesso às políticas públicas.

Afinal, como as equipes de saúde lidam com a hierarquia imposta com suas diferenças e configurações sociais específicas?

Diante da questão exposta, entendemos que a situação deve ser encarada com a importância que lhe é apropriada, uma vez que as relações estabelecidas entre população e profissionais de saúde podem se declarar, de acordo com Brasil ${ }^{7}$, instáveis e intensificadas pelo despreparo dessas equipes para lidar com a perspectiva subjetiva nas práticas de atenção.

As equipes de saúde, em seu trabalho vivo em ato, de acordo com Merhy ${ }^{8}$, enfrentam uma diversidade social em sua relação dialógica com a população adscrita. Ou seja, o conjunto de diferenças e valores compartilhados pelos indivíduos no espaço social, visando ao princípio de territorialização das ações, surge como mais um elemento na relação entre equipe e população.

Para Merhy ${ }^{8}$, tal descoberta possibilita a abertura de uma janela de discussão sobre a subjetividade do cuidado de saúde. Assegurar tal dimensão favorece um olhar mais atento às manifestações que ladeiam o trabalho em saúde, tal como a produção do cuidado.

Em conformidade com Laroc e Luccas ${ }^{9}$, essas especificidades passam a permear as relações que se estabelecem entre os atores sociais dentro de um determinado território. Adicionalmente, segundo os mesmos autores, podem provocar distorções no julgamento de quem observa o território de saúde considerando apenas o aspecto biologicista e antropocêntrico?. 
Além disso, perde-se, desse modo, a oportunidade de alcançar uma melhor compreensão das relações existentes nos territórios; isto é, peculiaridades que podem ser camufladas por outras mais acentuadas, como cor/raça e classe social. Assim, o entendimento de território por parte das equipes técnicas e usuários do Sistema Único de Saúde pode influenciar a forma como as ações serão implementadas e como esse território será incorporado a essas ações.

A dinâmica centrada na concepção de território, no que se refere à responsabilidade da Equipe de Saúde da Família (Brasil, 1994), trata da organização do então Programa ${ }^{10}$ que recomendava a cobertura por cada equipe de uma área geográfica abrangendo entre 800 e 1.000 famílias. Já em Brasil ${ }^{11}$, o conceito de adscrição modifica-se. A recomendação passa a ser que cada equipe deve se responsabilizar por uma área englobando entre 600 e 1.000 famílias. Para além dessa composição, o documento que reorienta o modelo assistencial da Estratégia Saúde da Família adverte do mesmo modo para a necessidade de se considerar a diversidade sociopolítica, econômica, entre outros aspectos da área.

A diversidade sociopolítica figura entre as necessidades de saúde de um determinado território, de forma similar ao estabelecimento das relações de poder, dependência e exclusão efetiva. Para Minayo ${ }^{12}$ :

E também reconhecer em uma sociedade específica como a disposição dessas relações pode implicar para a qualidade do atendimento e cobertura da política pública. Este conhecimento se configura como um grande desafio para a resolutividade dos problemas de saúde de uma população cada dia mais complexa e exigente de uma atuação melhorada e ampliada em diversos aspectos.

Nessa linha, a perspectiva promovida por Elias e Scotson ${ }^{4}$ soma-se a outros enfoques, inclusive o epidemiológico, tornando assim a leitura da realidade mais próxima da complexidade que ela mesma institui.

AS CONFIGURAÇÕES SOCIAIS ESPECÍFICAS E TERRITORIALIZAÇÃO EM SAÚDE

Diante das possibilidades de se pensarem e repensarem diferentes ângulos e perspectivas para a utilização de análises quantitativas, Barreto ${ }^{13}$ oferece relevantes ponderações. Para ele, os indivíduos não podem ser reduzidos a uma definição que envolva apenas aspectos biológicos. Subsiste o que denomina de profunda e complexa dialética multidimensional, em que biologia, espaço e tempo são interpretados como algo múltiplo, suplantando a simples soma destes. Para o autor, eventos da má saúde populacional são variações que, mesmo percebidas no nível individual (plano biológico/psíquico), configuram-se de forma convergente, tendo como consequência a estruturação das sociedades.

Essa pluralidade dos sujeitos, da qual Barreto ${ }^{13}$ aproxima-se, favorece laços complexos, que se unem e caracterizam uma desigualdade socioespacial. Tamanha diversidade não consegue ser expressa meramente em análises estatísticas e quantitativas.

No que se refere a esses limites, Barreto ${ }^{13}$ alerta que, na busca pela saúde, tal esforço não deve se ater a um processo isolado, mas imbricado a uma complexa trama estabelecida e interdependente da questão econômica, cultural, informacional.

A argumentação de Barreto ${ }^{13}$ entra em conformidade com Elias e Scotson ${ }^{4}$, quando estes afirmam que as análises quantitativas, quando utilizadas dissociadas de outras leituras, são inseridas em um método que restringe seu uso a uma concepção das sociedades como amontoados de pessoas, como populações estatísticas, o que desencadeia o desvio do foco das configurações sociais específicas da população. Diferenças profissionais ou de poder aquisitivo, por exemplo, não são suficientes para explicar a disparidade de status em um determinado território.

Consideramos, portanto, que, ao se pesquisarem os determinantes sociais da saúde, é impossível chegar a uma dedução através de uma mera análise quantitativa. Elias e Scotson ${ }^{4}$ apontam ainda que, por maior que seja o número de correlações estatísticas que se deseje estabelecer nas sociedades, elas não podem por si só levar a uma compreensão clara da forma como as configurações sociais específicas funcionam e afetam a população residente.

Elias e Scotson ${ }^{4}$ sustentam ainda que há um desafio de se ficar atento aos processos de investigação social, trazendo a atenção para o fato de que, ao descobrirmos movimentos, fluxos, descontinuidades, ficamos extasiados diante do novo, verificamos também que nos faltam conceitos e até palavras que permitam indicar uma aproximação adequada ao que está diante dos nossos olhos, ou seja, a convivência entre seres humanos inseridos em uma comunidade não apresenta equilíbrio ou harmonia ${ }^{4}$.

Nessa perspectiva, Helman ${ }^{14}$ relata que cada indivíduo tem seu próprio modo de explicar e tratar a má saúde, e os agentes de cura em cada grupo são organizados segundo regras de condutas, obedecendo a normas de admissão e critérios de relacionamento com seus pacientes/usuários. São as chamadas subculturas de cuidados de saúde.

Conquanto, para Elias e Scotson ${ }^{4}$, em alguns casos, tanto as diferenças qualitativas quanto as relações quantitativas são extremamente úteis como indicadores sociais. Certamente, é significativo o fato, a título de exemplo, de se identificar por meio da investigação epidemiológica o número de casos de determinada doença e sua relação a certo tipo de hábito alimentar. Ainda assim, uma observação afinada com a perspectiva de Elias e Scotson pode revelar que a complexidade existente, relacionada aos diferentes hábitos alimentares de determinada população, só pode ser apresentada de forma satisfatória se for explicada por símbolos verbais.

Elias e Scotson ${ }^{4}$ atentam que, sem o uso das palavras como instrumento de pesquisa, os números são mudos. Os diversos 
papéis que os grupos exercem diante de seus hábitos alimentares, adotados em suas diferentes configurações, podem ser considerados com um bom exemplo.

Muitas vezes, a utilização atual das estatísticas parece implicar que, quanto maiores às dimensões numéricas, maior a importância. No caso das minorias de Winston Parva, como em muitos outros, a significação sociológica de modo algum era idêntica à significação estatística. Elas apontavam para um fato conhecido de outras pesquisas, ainda que, talvez, não suficientemente salientado: o de que os dados sociais podem ser sociologicamente significativos sem ter significação estatística e podem ser estatisticamente significativos sem ter significação sociológica ${ }^{4}$.

Deste modo, para avançarmos no processo intencional de mudanças estruturais do SUS, faz-se necessário situá-las nos debates, objetivando uma melhor interpretação dessas configurações sociais e espaciais tão singulares.

Para adentrarmos nos princípios pressupostos da territorialização em saúde, nós nos ocuparemos do conceito de Nunes ${ }^{15}$, que afirma ser a noção sociopolítica de território em saúde a que mais segue em conformidade com as demandas de análise das ciências sociais e humanas. $\mathrm{O}$ autor afirma que só é possível tratar de demarcação ou delimitação se houver condições de se caracterizar uma pluralidade dos sujeitos.

Elias e Scotson ${ }^{4}$, ainda que não tenham como foco propriamente o debate saúde e território, permitem que possamos aplicar novos parâmetros para requalificar essa discussão. Os autores destacam a problemática do relacionamento entre indivíduos em uma comunidade e identificam e elucidam as relações de poder, dependência e exclusão existentes em uma sociedade específica e suas implicações em todos os níveis de sua organização, bem como na totalidade dos aspectos da vida dos habitantes ${ }^{4}$. A noção de espaço territorial, portanto, constitui categoria fundamental na perspectiva de ambos os teóricos.

Eles utilizam análise e sinopse das chamadas configurações, indo além das análises quantitativas e estatísticas sobre o território. Assim, apreendem a existência de grupos e subgrupos que, de outra forma, não se destacariam, atingindo então uma melhor compreensão das relações sociais existentes.

Elias e Scotson ${ }^{4}$ apresentam uma concepção que pretende eliminar a forma rígida do pensamento que diferencia e isola o indivíduo da sociedade. Para ambos, a análise de uma configuração social não pode ser restringida à análise de um elemento isoladamente. Não basta a compreensão de aspectos do comportamento ou das ações dos indivíduos, singularmente consideradas. É preciso avaliar a interdependência para as configurações que as pessoas estabelecem umas com as outras ${ }^{4}$.

O contexto analisado por Elias e Scotson ${ }^{4}$ refere-se a um território industrial urbano do centro da Inglaterra, envolvendo três subgrupos da mesma comunidade que se distinguem basicamente por uma disparidade de antiguidade no local entre os grupos estabelecidos e os recém-chegados (outsiders).
Apropriados dessas informações a respeito dos três subgrupos de uma mesma comunidade, os autores empenharam-se em descobrir quais recursos de poder favoreciam a afirmação da superioridade e o estigma de um grupo sobre outro.

Ressalta-se que o grupo autodenominado como "estabelecidos" tende a se identificar como pessoas "melhores", produzindo uma autoimagem de superioridade prontamente entendida, aliás, como superioridade humana. No entanto, por meio de uma observação mais esquadrinhada, pode-se, com certa frequência, descobrir um relevante padrão de coesão entre os estabelecidos quando comparados aos outsiders. Essa coesão sustenta-se pelo processo de estigmatização e erguimento de uma barreira emocional do grupo estabelecido para com o grupo outsider ${ }^{4}$.

O estudo das configurações sociais específicas ${ }^{4}$ apresenta-se como importante contribuição nos debates sobre saúde coletiva quando tais aspectos - pertencimento, coesão grupal, autopercepção - constituem pontos comuns numa imensa escala e passam a impor dificuldade de acesso às políticas públicas, inclusive sob a perspectiva da Saúde da Família ${ }^{4}$.

Geertz $^{16}$, afinado com a concepção de Elias e Scotson ${ }^{4}$ em relação às configurações sociais específicas das coletividades, afirma que o indivíduo pode ser um completo enigma para outro ser humano. Relata o exemplo da chegada de um indivíduo a um país estranho: "[...] com tradições inteiramente estranhas e [...] mesmo que se tenha um domínio total do idioma do país, nós não compreendemos o povo (e não por não compreender o que eles falam entre si). Não nos podemos situar entre eles"16(p. 9).

Práticas sanitárias que não se comprometam em conhecer as singularidades e configurações sociais específicas dos territórios de saúde assemelham-se à condição de estrangeiro que, de acordo Max Weber ${ }^{17}$, mesmo falando o idioma desse território, não compreende sua teia de significados, que é sua própria cultura.

Para Teixeira ${ }^{18}$, o desafio maior para o enfrentamento de tantas questões encontra-se nas ações que circundam o planejamento em saúde. Para a autora, é preciso que, como prática social, as respostas aos problemas de saúde de grupos populacionais tão distintos estejam associadas às condições de vida, assim como à assimilação das representações sociais que envolvem o processo saúde-doença.

Essas questões são fundamentais posto que, para Elias e Scotson, haja certo estranhamento sobre a ideia de se estudarem os indivíduos como princípios isolados de suas configurações. Para eles, quando se estabelece o pressuposto de que somente métodos estatísticos oferecerão as respostas aos problemas da sociedade, confundem-se profundamente as pesquisas sobre tais configurações ${ }^{4}$.

O pensamento de Elias e Scotson ${ }^{4}$ apresenta-se como substancial ferramenta para uma melhor percepção dos desafios e das possibilidades de atuação dos profissionais nos territórios de saúde, principalmente no que se refere à noção de território, 
que impõe desafios tanto para a gestão da Atenção Primária à Saúde quanto para a assistência do cuidado.

Os autores, ao se utilizarem da análise e da sinopse das configurações sociais específicas, divisam para além das pesquisas quantitativas e estatísticas. $\mathrm{O}$ caminho trilhado por eles segue uma lógica voltada para a necessidade de ir além da observação de um elemento isolado. Para ambos é primordial que decorra uma análise das configurações sociais. Estas, por seu turno, devem ser compreendidas através dos aspectos do comportamento ou das ações dos indivíduos, pois configurações sociais singularmente consideradas revelam-se insuficientes em termos analíticos. É preciso pensar a interdependência das configurações que as pessoas estabelecem umas com as outras, portanto.

No que tange a essa discussão e diante de um contexto marcado pela fragmentação do processo de trabalho, pelo precário entendimento entre profissionais e população, além do despreparo das equipes em lidar com o aspecto subjetivo nas práticas do cuidado; o referencial teórico de Elias e Scotson, acerca das configurações sociais específicas, traz importante contribuição, inclusive com implicações para a qualidade do atendimento e a cobertura da política pública de Saúde.
Nesse âmbito, a não utilização de instrumentos que incorporem as representações sociais que envolvem o processo saúde-doença acarreta um empobrecimento tanto nas reflexões como, posteriormente, nas ações a serem implantadas nos grupos sociais como objetos de estudo.

Elias e Scotson ${ }^{4}$ afirmam que diversas áreas de problemas sociologicamente relevantes continuam não sendo exploradas ou, quando exploradas, apresentam-se com a pecha de "meramente descritivas” por não configurarem estatísticas.

Entretanto, no campo da Saúde Coletiva, vê-se que, em um plano geral, esse processo requer investimento na qualificação dos profissionais de saúde para lidar com a subjetividade dos sujeitos assistidos. Tal disposição manifesta-se como fomento para se reafirmar o propósito do Ministério da Saúde ao preconizar uma política permanente de assistência baseada nos princípios de integralidade e equidade nas ações.

Em uma perspectiva atenta sobre a Saúde Coletiva ser um campo fértil à questão da interdisciplinaridade, conceito defendido neste texto, as concepções de Elias e Scotson sobre as configurações sociais específicas, vêm reiterar, inclusive e especialmente para a impertinência de oposições quantidade e qualidade, comumente referidas por atores no campo e fora dele.

\section{REFERÊNCIAS}

1. Gondim GMM, Monken M, Rojas LI, Barcellos C, Peiter P. O território da saúde: a organização do sistema de saúde e territorialização. In: Miranda AC, Barcellos C, Moreira JC, Monken M. Território, ambiente e saúde. Rio de Janeiro: Fiocruz; 2008. p. 183-203.

2. Monken M, Barcellos C. Vigilância em saúde e território utilizado: possibilidades teóricas e metodológicas. Cadernos de Saúde Pública. 2005;21(3):898-906. http://dx.doi.org/10.1590/S0102-311X2005000300024.

3. Baralhas M, Pereira MA. Prática diária dos agentes comunitários de saúde: dificuldades e limitações da assistência. Rev Bras Enferm. 2013;66(3):35865. http://dx.doi.org/10.1590/S0034-71672013000300009. PMid:23887784.

4. Elias N, Scotson JL. Os estabelecidos e os outsiders: sociologia das relações de poder a partir de uma pequena comunidade. Rio de Janeiro: Jorge Zahar Editor; 2000.

5. Santos AL, Rigotto RM. Território e territorialização: incorporando as relações produção, trabalho, ambiente e saúde na Atenção Básica à Saúde. Trab Educ Saúde (Impresso). 2010;8:387-406.

6. Nascimento LJ, Sthephan C, Nunes E. Cientistas sociais da Saúde Coletiva: uma abordagem pela óptica fuzzy. Cien Saude Colet. 2015;20(5):1-11. PMid:26017960.

7. Ministério da Saúde. Secretaria de Atenção à Saúde. Humaniza SUS: documento base para gestores e trabalhadores do SUS [Internet]. 4. ed. Brasília: Núcleo Técnico da Política Nacional de Humanização; 2010 [citado em 2016 Mai 9]. Disponível em: http://bibliotecadigital.puccampinas.edu.br/services/e-books/humanizasus_documento_gestores_ trabalhadores_sus.pdf

8. Merhy EE. Saúde: a cartografia do trabalho vivo. São Paulo: Hucitec; 2002.
9. Laroc LM, Luccas DS. Relações de poder no território de uma Unidade Básica de Saúde. Revista Saúde e Pesquisa. 2008;2(3):311-8.

10. Brasil. Programa de Saúde da Família (PSF). Brasília: Ministério da Saúde; 1994.

11. Brasil. Saúde da família: uma estratégia para reorientação do modelo assistencial. Brasília: Ministério da Saúde; 1997.

12. Minayo MCS. O desafio do conhecimento: pesquisa qualitativa em saúde. 2. ed. São Paulo: Hucitec; 1999.

13. Barreto ML. Papel da epidemiologia no desenvolvimento do Sistema Único de Saúde no Brasil: histórico, fundamentos e perspectivas. Rev Bras Epidemiol. 2002;5(Supl 1):4-17. http://dx.doi.org/10.1590/S1415790X2002000400003.

14. Helman CG. Cultura, saúde e doença. 2. ed. Porto Alegre: Artes Médicas; 1994. Eliane Mussnich, trad.

15. Nunes PHF. A influência dos recursos naturais na transformação do conceito de território. Questiones Constitucionales. Jul-Dic 2006;(15):69-112.

16. Geertz C. A interpretação das culturas. 1. ed. Rio de Janeiro: Editora Guanabara Koogan; 1989, p.9.

17. Weber M. Economía y sociedad. México: Fondo de Cultura Económica; 1984.

18. Teixeira CF. Epidemiologia e planejamento de saúde. Cien Saude Colet. 1999;4(2):287-303. http://dx.doi.org/10.1590/S1413-81231999000200005.

Recebido em: Jun. 07, 2018 Aprovado em: Jan. 26, 2019 